Research Article

\title{
Reverse Logistics Network Design of Electric Vehicle Batteries considering Recall Risk
}

\author{
Hao Hao $\mathbb{D}^{D}$, Yichen Sun $\left(\mathbb{D}\right.$, Xueyun Mei ${ }^{(D)}$, and Yanjun Zhou \\ School of Economics and Management, Shanghai Polytechnic University, Shanghai 201209, China \\ Correspondence should be addressed to Yichen Sun; sunyichen1013@163.com
}

Received 21 January 2021; Revised 1 March 2021; Accepted 9 August 2021; Published 19 August 2021

Academic Editor: Libor Pekař

Copyright (c) 2021 Hao Hao et al. This is an open access article distributed under the Creative Commons Attribution License, which permits unrestricted use, distribution, and reproduction in any medium, provided the original work is properly cited.

In 2018-2019, the recall scale of electric vehicles (EVs) in China reached 168,700 units; recalls account for approximately $6.9 \%$ of sales volume. There are imperative reasons for electric vehicle batteries (EVBs) recalls, such as mandatory laws or policies, safety and environmental pollution risks, and the high value of EVB echelon use, and thus, it has become increasingly important to reasonably design a reverse logistics (RL) network for an EVB recall. In this study, a multiobjective and multiperiod recall RL network model is developed to minimize safety and environmental risks, maximize the social responsibility and economic benefits, and consider the characteristics of EVBs, including the configuration of key recall facilities and the control of recall flows. The results of this study will help EVB practitioners, relevant departmental policymakers, and others to comprehensively understand the recall of EVBs, strengthen the safety and environmental protection issues in the EVB recall process, and promote the establishment of a safe, green, and sustainable EVB recall RL network.

\section{Introduction}

According to the International Energy Agency [1], global EV ownership exceeded 7.2 million units by the end of 2019, and the annual sales of EVs are predicted to reach 80 million units by 2030 in the context of sustainable development. A report released by China's General Administration of State Market Regulation [2] shows that 168,700 EVs were recalled in 2018-2019; recalls account for approximately $6.9 \%$ of sales volume. China's Ministry of Industry and Information Technology released a report on the Big Data Safety and Supervision Achievements of the National Supervision Platform for EVs [3], which showed that, from May to August 2019, 58\% of vehicles burned, where the cause of the fire was identified, were due to battery problems. Defective EVBs, product recalls, and accidents are common to current RL process triggers [4]. EVBs are different from ordinary products, and there are certain safety hazards in EVBs. There are serious environmental pollution hazards from the leakage of EVBs [5]. Therefore, their safety, environmental protection, and whether they can be effectively recalled and disposed of are also a wide concern. In the event of an emergency recall owing to safety or environmental concerns, it is even more important to establish a fast and safe RL network.

This study considers the safety risks of EVB recall such as spontaneous combustion and explosion as well as environmental pollution risks such as liquid leakage and corrosion and establishes an RL network for new energy vehicle EVBs based on recall risks. The aim of this study is to solve the optimization problem of network site selection during this safe and green recall process.

Firstly, this study constructs an RL network for EVB recall. This network includes service outlet, recall site, recall processing center, recall transfer center, and echelon use center. Second, based on fitting the existing data and predicting the possible recall of EVBs in the next three years, this study establishes a multiobjective and multiperiod recall RL network site selection model, which aims at minimizing safety and environmental risks and maximizing social responsibility and economic benefits.

In the study, the network considers innovation indicators such as testing cost, packaging cost, and recall time of EVBs, considers economic indicators such as transportation 
cost and storage cost, and considers social responsibility indicators such as employment opportunities. Finally, we determine the appropriate location and flow in the recall RL network.

The contributions of this study are as follows: (1) factor characteristics of EVB recalls, the costs of a safety risk detection and packaging when considering the potential environmental contamination, and job growth in terms of social responsibility are considered, (2) a mixed-integer nonlinear programming (MINLP) model with the goal of minimizing safety and environmental risks and maximizing social responsibility and economic benefits is established, and (3) sensitivity analysis is performed. The analysis examines the weighting of safety and environmental protection, social responsibility, and economic benefit and argues that the economic gains made are meaningful only if safety and environmental protection are ensured and that only by coordinating safety and environmental protection with economic benefits can we encourage the sustainable development of an EVB recall RL network.

The remainder of this paper is organized as follows. Section 2 provides a brief review of the related literature. The methodology employed in this study is described in Section 3. Assumptions and formulas are presented in Section 4. Section 5 describes an empirical study conducted on the RL network of Shanghai's EVB recall when considering the risk of such a recall. Finally, Section 6 provides some concluding remarks and discusses the limitations of this research.

\section{Literature Review}

A review of the concept and development of RL: Stock [6] first proposed the concept of RL, which is broadly defined to include all logistics activities related to resource conservation, recycling, replacement, reuse of materials, and disposal. To address the social sustainability of RL, Sarkis et al. [7] studied RL from the triple bottom line of economic, environmental, and social benefits and established sustainability indicators related to various RL practices. Whereas RL has been considered a cost for companies in the past, Hao et al. [8] argued that, in the global trend of the green low-carbon cycle, RL is an important way for companies to generate additional profits. Thus, Wang et al. [9] found that improving the efficiency of end-of-life EV RL is an important way to promote green development and social benefits. Alamerew and Brissaud [4] expanded the thinking regarding $\mathrm{RL}$, moving from a resource-efficient model to a fully circular and sustainable model.

In the study on RL network design, Fleischmann et al. [10] summarized the common features of RL networks, studied the location of RL network facilities and inventory management, and conducted a quantitative study of RL decision models. Barros et al. [11], Min et al. [12], Zhou and Cao [13], and Zaarour [14] all proposed MINLP models and considered using heuristic algorithms to optimize them. Governments have also started to gradually pay attention to the development of RL, and Zhou et al. [15] constructed a multiperiod multiobjective dynamic MINLP model by optimizing the social cost, economic cost, and recovery revenue based on government subsidies and solved it using the particle swarm algorithm. The green environment has been an integral part of a logistics network design; in forward logistics, Zhen [16] developed a multiperiod green supply chain network and established the dual-objective model with the aim of minimizing total supply chain costs and carbon emission; in RL, Govindan et al. [17], Trochu et al. [18], and Nageswara et al. [19] took environmental policy requirements, pollution reduction, and carbon emission reduction into consideration to develop a rational RL network and established the MINLP model. Most recent studies have used the MINLP method to model RL networks, and increasing focus has been given to green and low-carbon issues in the models; however, to date, little attention has been paid to safety.

Regarding the optimization of an EVB RL network, Hoyer et al. [20] developed a strategic framework for the design of recycling networks for waste lithium-ion batteries of EVs. Hao et al. [21] evaluated the feasibility of RL of endof-life vehicle batteries based on an improved fuzzy neural network. EVBs are different from ordinary products. Huo et al. [22] argued that EVBs have certain corrosive, flammable, explosive, and toxic properties, and the unregulated recycling and disposal of EVBs may lead to fire, explosion, and pollution of the environment. In addition to the recall of EVBs, which has certain safety risks, Sloop et al. [23] studied the recall of lithium batteries and found that batteries can create a useful value after recall. Based on ecological, economic, and geostrategic reasons, Hakim et al. [24] designed a sustainable recycling network for lithium batteries under uncertainty and developed recycling network modeling and value chain solutions to match batteries. Wang et al. [25] argued the need for end-of-life lithium battery recycling management and the establishment of a sound end-of-life lithium battery infrastructure to reduce the uncertainty of $\mathrm{RL}$ and maximize the use of battery recycling to reduce economic costs. Jayant et al. [26] proposed a predictive approach that can calculate the battery collection costs, transfer time, transfer costs, and resource utilization and designed and developed a simulation model of an RL network using the example of waste battery collection in Sangrur District of northern India. In addition, Hendrickson [27] combined a life cycle assessment and GIS to analyze how the energy, greenhouse gas, water, and standards of a lithium-ion battery end-of-life infrastructure network affect air pollutants and determined the optimal location of battery disassembly and recycling facilities for use in a California recycling program. Tadaros et al. [28] proposed a discrete multiperiod facility location allocation RL MINLP model to address the recycling of EVBs from EVs in Sweden. In addition, Masudin et al. [29] developed an RL network for battery recycling from the end customer to the remanufacturing process from the perspectives of environment and transportation costs. Wang et al. [30] developed an "Internet+" battery recycling system with a reward and punishment mechanism to solve the problem of recycling endof-life batteries. From the above studies, it can be seen that many approaches have taken cost reduction, environmental issues, and uncertainty into consideration when designing 
EVB RL networks, and most have adopted the MINLP model as a solution.

A product recall is an important element of RL [31]. However, there have been few studies on recall RL network optimization. Jayaraman et al. [32] established an RL network for product recycling, product recall, product disposal, and hazardous product recycling with the goal of cost minimization. Hu et al. [33] developed a model of a closedloop supply chain network for recall with the MINLP model, taking into account uncertainty in demand, uncertainty in the number of recalls and returns, the e-commerce environment, and the time value of money. Chen et al. [34] developed a stochastic mixed-integer programming model of a CLSC network with the goal of minimizing the total cost and the total recall handling response time, based on the urgency of product recalls and the management characteristics of recall emergency management. Pan et al. [35] proposed that recall management is essentially a special type of RL link and developed an improved genetic algorithmbased model for the location and allocation of recall centers based on the characteristics of a food recall network. Pharmaceuticals, as one of the items with the greatest impact on human health, have common features with EVBs in terms of time urgency, and Gao [36] and Huang and Wang [37] established an MINLP model for drug recalls with the goal of achieving the minimum cost and time based on the characteristics of multiple levels, multiparty correlation, contingency, and high costs of a drug recall. RL networks are solved using a heuristic algorithm.

To summarize, from the above studies, it can be seen that the methods used for RL network optimization are mainly based on an MINLP. Single- and multiobjective methods have been constructed; single and multiple periods have been considered. Overall, the existing research is mainly based on multiple objectives and periods for RL networks. In the future, recall RL network design research will pay more attention to being green, environmental protection, and safety.

The above-mentioned studies have the goal of minimizing costs, maximizing the economy, and minimizing time [38] as well as minimizing carbon emissions. However, there has been little research on RL for the recall of EVBs in EVs with the goals of minimizing safety and environmental risks and maximizing social responsibility and economic benefit.

In recent years, EVBs have become a research hotspot, although little research has been conducted on the design of RL networks for recalls with EVBs as the subject of study. The development of a rational recall RL network is critical to the rapid and safe recall of faulty EVBs.

Therefore, considering the urgency of the real-life problem, and there is still a certain gap in the research on EVBs recall. In this paper, we consider the safety and environmental risks, social responsibility, and economic benefits as the goal, comprehensively consider various factors such as an RL recall time, testing costs, packaging costs, site setup scale, transportation costs, operating costs, the number of jobs created, and storage costs, and collect 364 EVB service outlets in Shanghai to build a multiobjective multiperiod MINLP model that considers the recall risk to determine the location and number of each RL facility and the flow and direction of the RL of an EVB recall for each facility.

\section{Methodology—Fourier Model}

This study will use the Fourier prediction model to forecast the sales volume of new energy vehicles.

According to the definition of the Fourier series [39, 40], the periodic function $f(x)$ can be described by a linear combination of trigonometric functions. If the period of $f(x)$ is $T$, the angular frequency $\Omega=2 \pi / T$, and the frequency $f=1 / T$. The expression for the Fourier series expansion is

$$
\begin{aligned}
f(x)= & a_{0}+a_{1} * \cos (x * \Omega)+b_{1} * \sin (x * \Omega)+a_{2} \\
& * \cos (2 x * \Omega)+b_{2} * \sin (2 x * \Omega) .
\end{aligned}
$$

Fourier model prediction is calculated to find the best result under the minimum variance estimate of the prediction error. The geometric description of the best prediction principle based on the Fourier model is shown in Figure 1 . In order to minimize the prediction error $e_{t}(l)$, the best prediction $x_{t}(l)$ must be the orthogonal projection of the vector $x_{t+l}$ in the three-dimensional space.

The Fourier prediction model will be fitted and predicted using the cftool toolbox inside Matlab 2018a for the relevant data.

\section{Recall Reverse Logistics Model}

4.1. Problem Definition. The objective of this study is to design an RL network for EVBs that considers the risk of recall. The network setup testing cost indexes consider the safety risk of an EVB recall, and packaging cost indices consider the environmental pollution risk of an EVB recall and establish an RL infrastructure for an EVB recall that includes service outlets, recall sites, recall processing centers, recall transfer centers, and echelon use centers. As shown in Figure 2, EVBs are recalled from the owners at the service outlets for a short period of storage and then transported in batches to the recall processing center. After sorting, picking, testing, and packaging at the recall processing center, the EVBs will be shipped to the recall transfer center and consolidated to the echelon use center. At the recycling center, the recalled EVBs will be sorted into different categories for recycling, parts reuse, material reuse, or resource treatment.

In this study, a multiobjective and multiperiod MINLP model is established based on the characteristics of the EVBs and considers the recall risk to achieve the coordinated development of safety and environmental benefits and economic benefits, providing a reference for the management decisions of relevant personnel.

4.2. Model Assumptions. The assumptions of the proposed model are as follows: 


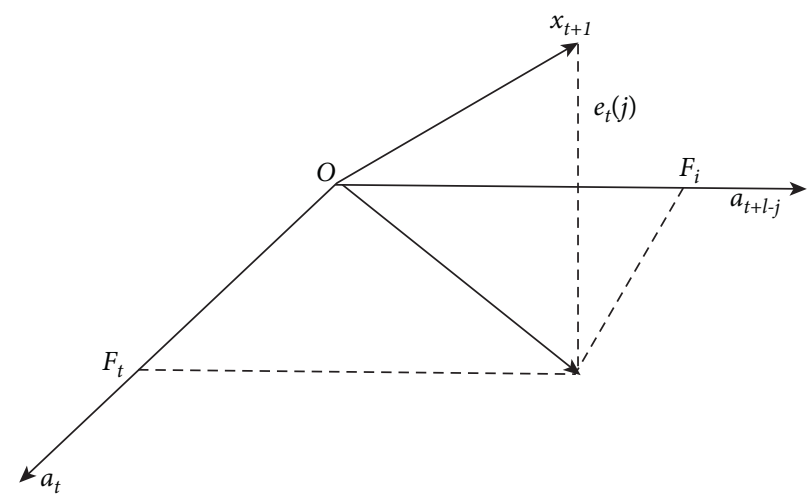

FIGURE 1: Geometric representation of the best prediction.

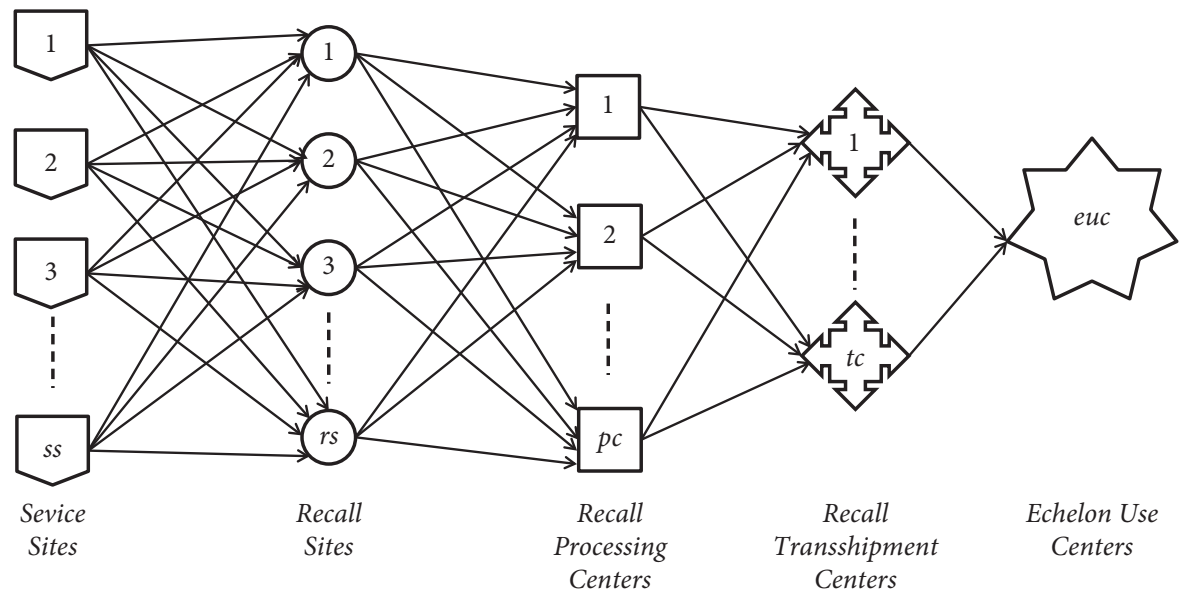

Figure 2: Recall RL network for EVBs.

(1) All EVBs are collected through service outlets to recall sites, and there will be no direct delivery of EVBs to recall sites by vehicle owners or EVB owners.

(2) Recall processing centers, recall transfer centers, and echelon use centers obey an even distribution of storage costs.

(3) The recall sites, recall processing centers, recall transfer centers, and echelon use centers are not engaged in any acts or events that damage the EVBs during storage.

(4) At the end of the operating period, the recall sites send all EVBs to the recall processing center.

(5) To ensure the reliability of the recall network and its stable operation in the case of an emergency, it is assumed that all EVBs of the recalled EVs will need to be collected for test, repair, disposal, echelon use, or resource treatment.

\subsection{Model Formulation}

4.3.1. Sets. In this section, the collection, model parameters, and decision variable symbols are described, and the proposed multiobjective multiperiod MINLP model is configured using the mentioned components.

$R$ : set of recall sites, index by $r$

$S$ : set of recall processing centers, index by $s$

$T$ : set of recall transfer centers, index by $t$

$E$ : set of echelon use centers, index by $e$

$P$ : set of Period, index by $p$

\subsubsection{Parameters}

$f i_{s}^{p}$ : fixed input cost of establishing a recall processing center at the alternative site $s$ in period $p$

$f i_{t}^{p}$ : fixed input cost of establishing a recall transfer center at the alternative site $t$ in period $p$

$f i_{e}^{p}$ : fixed input cost of establishing an echelon use center at the alternative site $e$ in period $p$

$j c_{s}^{p}$ : number of jobs created in the recall processing center $s$ in period $p$

$j c_{t c}^{p}$ : number of jobs created in the recall transfer center $t$ in period $p$

$j c_{e}^{p}$ : number of jobs created in the echelon use center $e$ in period $p$ 
sc: unit transportation costs per EVB

$p k c$ : unit packaging cost per EVB

$d c$ : unit testing cost per EVB

$w c_{s}$ : storage cost per unit per day at the recall processing center $s$

$w c_{t}$ : storage cost per unit per day at the recall transfer center $t$

$w c_{e}$ : storage cost per unit per day at the echelon use center $e$

$o c_{s}$ : unit operating costs of the recall processing center $s$ $o c_{t}$ : unit operating costs of the recall transfer center $t$ $o c_{e}$ : unit operating costs of echelon use center $e$

$l c_{s}$ : daily labor cost per employee at the recall processing center $s$

$l c_{t}$ : daily labor cost per employee at the recall transfer center $t$

$l c_{e}$ : daily labor cost per employee at echelon use center $e$

$d t_{r s}$ : distance from the recall sites $r$ to the recall processing center $s$

$d t_{s t}$ : distance from the recall processing center $s$ to the recall transfer center $t$

$d t_{t e}$ : distance from the recall transfer center $t$ to the echelon use center $e$

$M_{s}$ : maximum processing capacity of the recall processing center $s$

$M_{t}:$ maximum processing capacity of the recall transfer center $t$

$M_{e}$ : maximum processing capacity of echelon use center $e$

$W_{s}^{p}$ : maximum inventory capacity of recall processing center $s$

$W_{t}^{p}$ : maximum inventory capacity of recall transfer center $t$

$W_{e}^{p}$ : maximum inventory capacity of echelon use center e

$L_{s}$ : maximum construction of recall processing center $s$

$L_{t}$ : maximum construction of recall transfer center $t$

$L_{e}$ : maximum construction of echelon use center $e$

$Q_{f}$ : predicted number of recalls for EVBs

Pr: value generated after echelon use of EVBs (after costs)

$\beta$ : utilization rate of echelon use center $e, 0 \leq \beta \leq 1$

$\delta_{1}, \delta_{2}$ : discount cut-off point for transport size

$\gamma_{1}, \gamma_{2}$ : penalty cut-off point for transport distance

$f\left(T_{r 0}, d t_{r 0}\right)=s c \cdot \delta \gamma$. Here, $\delta$ represents the discount rate, depending on the amount of transport $T_{r s}^{p}$ between the recall site $r$ and the recall processing center $s$. In addition, $\gamma$ represents the penalty rate, depending on the time to recall of the EVB to be recalled to recall site $r$, where time is replaced by distance, depending on the distance $d t_{r s}$ between the recall site $r$ and the recall processing center $s$.

$\delta= \begin{cases}\delta_{0}, & \text { for } T_{r i} \leq k_{1}, \\ \delta_{1}, & \text { for } k_{1}<T_{r i} \leq k_{2} \\ \delta_{2}, & \text { for } T_{r i}>k_{2}\end{cases}$

$\gamma= \begin{cases}\gamma_{0}, & \text { for } d t_{r i} \leq g_{1}, \\ \gamma_{1}, & \text { for } g_{1}<d t_{r i} \leq g_{2}, \\ \gamma_{2}, & \text { for } d t_{r i}>g_{2} .\end{cases}$

4.3.3. Variables

$Y_{r s}^{p}=\left\{\begin{array}{ll}1, & \text { recall sites } r \text { assigned to recall processing center } s \text { in period } p, \\ 0, & \text { otherwise }\end{array} ;\right.$

$Y_{s t}^{p}= \begin{cases}1, & \text { recall processing center } s \text { is assigned to recall transfer center } t \text { in period } p, \\ 0, & \text { otherwise }\end{cases}$

$Y_{t e}^{p}= \begin{cases}1, & \text { recall transfer center } t \text { is assigned to echelon use center } e \text { in period } p, \\ 0, & \text { otherwise }\end{cases}$

$Y_{s}^{p}= \begin{cases}1, & \text { recall processing center } s \text { is established in period } p, \\ 0, & \text { otherwise }\end{cases}$

$Y_{t}^{p}= \begin{cases}1, & \text { recall transfer center } t \text { is established in period } p, \\ 0, & \text { otherwise }\end{cases}$

$Y_{e}^{p}= \begin{cases}1, & \text { echelon use center } e \text { is established in period } p, \\ 0, & \text { otherwise }\end{cases}$

$T_{r s}^{p}$ : the number of EVBs transported from the recall sites $r$ to the recall processing center $s$ in period $p$;

$T_{s t}^{p}$ : the number of EVBs transported from the recall processing center $s$ to the recall transfer center $t$ in period $p$;

$T_{t e}^{p}$ : the number of EVBs transported from the recall transfer center $t$ to the echelon use center $e$ in period $p$.

4.4. Objective Functions. In this study, the three objectives are divided into two parts. The first part is the level of safety and environmental responsibility, including subobjective one (SET) and subobjective two (SR). The second part is the level of economic benefit, including subobjective three (EB). In this study, we integrate the safety and environmental responsibilities and economic benefits to maximize the overall benefits. Equation (2) is as follows:

$$
\max F=(\mathrm{SR}-\mathrm{SET})+\mathrm{EB} .
$$

Subobjective 1. Minimize safety and environmental risks, minimize response time

$$
\begin{aligned}
\mathrm{SET}= & \sum_{p} \sum_{s} \sum_{t} d t_{s t} \cdot Y_{t}^{p}+\sum_{p} \sum_{t} \sum_{e} \frac{Y_{e}^{p}}{M_{e}^{p} \cdot d t_{t e} \cdot \beta} \\
& +\sum_{p} \sum_{r} \sum_{s} d t_{r s} \cdot Y_{s}^{p} .
\end{aligned}
$$

Subobjective 2. Maximize social responsibility

$$
\mathrm{SR}=\sum_{p}\left(\sum_{s} j c_{s}^{p} \cdot Y_{s}^{p}+\sum_{t c} j c_{t}^{p} \cdot Y_{t}^{p}+\sum_{e u c} j c_{e}^{p} \cdot Y_{e}^{p}\right) .
$$

Subobjective 3. Maximize economic efficiency 
$\mathrm{EB}=\sum_{p} \sum_{e} W_{e}^{p} \cdot \operatorname{Pr} \cdot \beta-\mathrm{EC}$

$\mathrm{EC}=C_{1}+C_{2}+C_{3}+C_{4}+C_{5}+C_{6}+C_{7}$,

$C_{1}=\sum_{p}\left(\sum_{s} f i_{s}^{p} \cdot Y_{s}^{p}+\sum_{t} f i_{t}^{p} \cdot Y_{t}^{p}+\sum_{e} f i_{e}^{p} \cdot Y_{e}^{p}\right)$,

$C_{2}=\sum_{p} \sum_{r} \sum_{s} s c \cdot d_{r s} \cdot T_{r s}^{p}+\sum_{p} \sum_{s} \sum_{t} s c \cdot d t_{s t} \cdot T_{s t}^{p}$

$+\sum_{p} \sum_{t} \sum_{e} s c \cdot d t_{t e} \cdot T_{t e}^{p}$

$C_{3}=\sum_{p} \sum_{r} \sum_{s} d c \cdot T_{r s}^{p}$

$C_{4}=\sum_{p} \sum_{r} \sum_{s} p k c \cdot T_{r s}^{p}$

$C_{5}=\sum_{p}\left(\sum_{s} w c_{s} \cdot W_{s}^{p}+\sum_{t} w c_{t} \cdot W_{t}^{p}+\sum_{e} w c_{e} \cdot W_{e}^{p}\right)$,

$C_{6}=\sum_{p}\left(\sum_{s} j c_{s}^{p} \cdot l c_{s}+\sum_{t} j c_{t}^{p} \cdot l c_{t}+\sum_{e} j c_{e}^{p} \cdot l c_{e}\right)$,

$C_{7}=\sum_{p} \sum_{r} \sum_{s} o c_{s} \cdot T_{r s}^{p}+\sum_{p} \sum_{s} \sum_{t} o c_{t} \cdot T_{s t}^{p}+\sum_{p} \sum_{t} \sum_{e} o c_{e} \cdot T_{t e}^{p}$

Equation (2) indicates the optimal value of recall RL network. Equation (3) indicates that the recall of EVBs should ensure the safety of the city and reduce environmental pollution, and EVBs with potential problems should be recalled within the shortest possible time such that the risk of safety and pollution is minimized and the response time is the shortest. Here, $\sum_{p} \sum_{r} \sum_{s} d t_{r s} \cdot Y_{s}^{p}$ represents the distance between the recall processing center $s$ and the recall site $r$, $\sum_{p} \sum_{s} \sum_{t} d t_{s t} \cdot Y_{t}^{p}$ represents the distance between the recall transfer center $t$ and recall processing center $s$, and $\sum_{p} \sum_{t} \sum_{e} Y_{e}^{p} / W_{e}^{p} \cdot d t_{t e} \cdot \beta$ represents the size of the echelon use center $e$ and the distance to the recall transfer center $t$. Equation (4) indicates the maximization of the social responsibility of an enterprise that recalls the traction batteries of EVs, and social responsibility is expressed by the number of jobs created; in addition, $\sum_{s} j c_{s}^{p} \cdot Y_{s}^{p}$ represents the number of jobs created in the recall processing center, $\sum_{t} j c_{t}^{p} \cdot Y_{t}^{p}$ indicates the number of jobs created in the recall transfer center, and $\sum_{e} j c_{e}^{p} \cdot Y_{e}^{p}$ represents the number of jobs created in the echelon use center. Equation (5) indicates the economic benefits of the recall enterprise, and $W_{e}^{p} \cdot \operatorname{Pr} \cdot \beta$ represents the economic income of the recall enterprise. Equation (6) indicates the economic cost of the recall enterprise. Equation (7) represents the sum of the construction costs for each period, $\sum_{s} f i_{s}^{p} \cdot Y_{s}^{p}$ is the construction cost of the recall processing center, $\sum_{t} f i_{t}^{p} \cdot Y_{t}^{p}$ is the construction cost of the recall transfer center, and $\sum_{e} f i_{e}^{p} \cdot Y_{e}^{p}$ represents the construction cost of the echelon use center. Equation (8) indicates the sum of transport costs per period, $\sum_{p} \sum_{r} \sum_{s} s c$. $d t_{r s} \cdot T_{r s}^{p}$ represents the cost of transportation from the recall site to the recall processing center, $\sum_{p} \sum_{s} \sum_{t} s c \cdot d t_{s t} \cdot T_{s t}^{p}$ is the cost of transportation from the recall processing center to the recall transfer center, and $\sum_{p} \sum_{t} \sum_{e} s c \cdot d t_{t e} \cdot T_{t e}^{p}$ represents the cost of transportation from the recall transfer center to the echelon use center. Equation (9) is the sum of the testing costs of the recalled EVBs. Equation (10) shows the sum of the packaging costs of the recalled EVBs. Equation (11) indicates the sum of the storage costs for each period, $\sum_{s} w c_{s} \cdot W_{s}^{p}$ represents the storage costs for the recall processing centers, $\sum_{t} w c_{t} \cdot W_{t}^{p}$ represents storage costs for the recall transfer centers, and $\sum_{e} w c_{e} \cdot W_{e}^{p}$ represents the storage costs for echelon use centers. Equation (12) indicates the sum of the labor costs for each period, $\sum_{s} j c_{s}^{p} \cdot l c_{s}$ represents the labor costs for the recall processing centers, $\sum_{t} j c_{t}^{p} \cdot l c_{t}$ represents the labor costs for the recall transfer centers, and $\sum_{e} j c_{e}^{p} \cdot l c_{e}$ represents the labor costs for echelon use centers. Equation (13) indicates the operating costs, $\sum_{p} \sum_{s} \sum_{t} o c_{s} \cdot T_{r s}^{p}$ is the operating costs for the recall processing centers, $\sum_{p} \sum_{s} \sum_{t} o c_{t} \cdot T_{s t}^{p}$ represents the operating costs for recall transfer centers, and $\sum_{p} \sum_{t} \sum_{e} o c_{e} \cdot T_{t e}^{p}$ is the operating costs for echelon use centers.

The above are subject to the following:

$$
\begin{gathered}
\sum_{s} Y_{r s}^{p}=1, \quad \forall r, p, \\
\sum_{t} Y_{s t}^{p}=1, \quad \forall s, p, \\
\sum_{e} Y_{t e}^{p}=1, \quad \forall t, p, \\
\sum_{s} T_{r s}^{p} \cdot Y_{s}^{p}=Q_{f}, \quad \forall r, p, \\
\sum_{s} T_{r s}^{p} \cdot Y_{s}^{p}=\sum_{t} T_{s t}^{p} \cdot Y_{t}^{p}, \quad \forall r, p, \\
\sum_{t} T_{s t}^{p} \cdot Y_{t}^{p}=\sum_{e} T_{t e}^{p} \cdot Y_{e}^{p}, \quad \forall s, p, \\
\sum_{s} T_{r s}^{p} \cdot Y_{s}^{p}=\sum_{t} W_{s}^{p} \cdot Y_{s}^{p}, \quad \forall r, p, \\
\sum_{r} T_{s t}^{p} \cdot Y_{t}^{p}=\sum_{e} W_{t}^{p} \cdot Y_{t}^{p}, \quad \forall s, p, \\
\sum_{e} T_{t e}^{p} \cdot Y_{e}^{p}=\sum_{s}^{p} W_{e}^{p} \cdot Y_{e}^{p}, \quad \forall t, p,
\end{gathered}
$$




$$
\begin{gathered}
\sum_{t} T_{t e}^{p} \cdot Y_{e}^{p} \leq M_{e}, \quad \forall e, p, \\
W_{s}^{p} \cdot Y_{s}^{p} \leq V_{s}, \quad \forall s, p, \\
W_{t}^{p} \cdot Y_{t}^{p} \leq V_{t}, \quad \forall t, p, \\
W_{e}^{p} \cdot Y_{e}^{p} \leq V_{e}, \quad \forall e, p, \\
1 \leq \sum_{s} Y_{s}^{p} \leq L_{s}, \quad \forall p, \\
1 \leq \sum_{t} Y_{t}^{p} \leq L_{t}, \quad \forall p, \\
1 \leq \sum_{c} Y_{e}^{p} \leq L_{e}, \quad \forall p, \\
T_{r s}^{p}, T_{s t}^{p}, T_{t e}^{p} \geq 0, \quad \forall r, s, t, e, p, \\
W_{s}^{p}, W_{t}^{p}, W_{e}^{p} \geq 0, \quad \forall r, s, t, e, p, \\
Y_{r s}^{p}, Y_{s}^{p}, Y_{t}^{p}, Y_{e}^{p} \in\{0,1\}, \quad \forall r, s, t, e, p .
\end{gathered}
$$

Constraint (14) indicates that, in period $p$, there is a corresponding processing center $s$ for each recall site $r$. Constraint (15) indicates that, in period $p$, each selected recall processing center $s$ has a corresponding recall transfer center $t$. Constraint (16) shows that, in period $p$, each selected recall transfer center $t$ has a corresponding echelon use center $e$. Constraint (17) indicates that, in period $p$, the number of EVBs transported from the recall sites to the recall processing centers is equal to the number of recalls recovered from the recall sites. Constraint (18) shows that, in period $p$, the number of EVBs transported from the recall sites to the recall processing centers is equal to the number of EVBs transported from the recall processing centers to the recall transfer centers. Constraint (19) indicates that, in period $p$, the number of EVBs transported from the recall treatment centers to the recall transfer centers is equal to the number of EVBs transported from the recall transfer centers to the echelon use centers. Constraint (20) shows that, in period $p$, the number of EVBs transported from the recall sites to the recall processing centers is equal to the number of EVBs in stock at the recall processing centers. Constraint (21) indicates that, in period $p$, the number of EVBs transported from the recall processing centers to the recall transfer centers is equal to the inventory level of the recall transfer centers. Constraint (22) shows that, in period $p$, the number of EVBs transported from the recall transfer centers to the echelon use centers is equal to the inventory level of the echelon use centers. Constraint (23) indicates that, in period $p$, the number of EVBs transported from the recall sites to the recall processing center does not exceed the maximum processing capacity of the recall processing center. Constraint (24) shows that, in period $p$, the number of EVBs transported from the recall
TABLE 1: Sales of EVs in Shanghai.

\begin{tabular}{cc}
\hline Year & Sales \\
\hline 2011 & 12 \\
2012 & 298 \\
2013 & 514 \\
2014 & 11271 \\
2015 & 47446 \\
2016 & 45474 \\
2017 & 61354 \\
2018 & 73724 \\
\hline
\end{tabular}

processing centers to the recall transfer center does not exceed the maximum processing capacity of the recall transfer center. Constraint (25) indicates that, in period $p$, the number of EVBs transported from the recall transfer centers to the echelon use centers does not exceed the maximum processing capacity of the echelon use centers. Constraint (26) shows that, in period $p$, the number of EVBs at the recall transfer center does not exceed the maximum inventory of the recall processing center. Constraint (27) indicates that, in period $p$, the number of EVBs in the recall transfer center does not exceed the maximum inventory of the recall transfer center. Constraint (28) shows that, in period $p$, the number of EVBs in the echelon use center does not exceed the maximum inventory in the echelon use center. Constraint (29) indicates that, in period $p$, the number of alternatively established recall processing centers does not exceed the maximum set number of recall processing centers. Constraint (30) shows that in period $p$, the number of alternatively established recall transfer centers does not exceed the maximum set number of recall transfer centers. Constraint (31) indicates that, in period $p$, the number of alternatively established echelon use centers does not exceed the maximum set number of echelon use centers. Finally, constraints (32) and (33) indicate that the variable is nonnegative, and constraint (34) shows that the guaranteed decision variable is 0 or 1 .

\section{Computational Results}

In this section, a case study of Shanghai EVBs is first described, and the calculation results are then presented.

5.1. Case Study. This study takes the Shanghai EVB recall as an example and establishes an RL network based on the recall volume of EVBs during the next three years. The locations of RL infrastructure such as service outlets, recall sites, recall processing centers, recall transfer centers, and echelon use centers were identified, and the flow directions were determined.

Table 1 shows the sales volume of EVs in Shanghai during the past years, as released by the vehicle management department of the Shanghai public security organs.

Hao et al. [41] forecasted the number of end-of-life vehicles using a hybrid model based on grey model and artificial neural network. This study is based on the actual 
sales volume of EVs in Shanghai from 2011 to 2018, as shown in Table 1, the Fourier transformation $[39,40]$ is used to fit the merger prediction, the functional relationship is shown in Figure 3 and equation (34), and the predicted sales volume of EVs from 2019 to 2021 is shown in Table 2.

$$
\begin{aligned}
f(x)= & a_{0}+a_{1} * \cos (x * \Omega)+b_{1} * \sin (x * \Omega)+a_{2} \\
& * \cos (2 x * \Omega)+b_{2} * \sin (2 x * \Omega) .
\end{aligned}
$$

The correlation coefficients are as follows:

$$
\begin{aligned}
& a_{0}=51200, \\
& a_{1}=-919.3, \\
& a_{2}=-3835, \\
& b_{1}=49070, \\
& b_{2}=-11200, \\
& w=0.4253 .
\end{aligned}
$$

According to the Matlab 2018a test, under a 95\% confidence interval, as shown in Table 3, R-square tends toward 1 , indicating that the Fourier function has a good fit.

According to equation (35), the sales volume of EVs in Shanghai from 2019 to 2021 is calculated as shown in Table 2.

According to the information released by the Ministry of Industry and Information Technology of China on EVB recycling service outlets, as shown in Table 4, there are 364 service outlets in Shanghai (as of July 20,2020), and only the Huangpu District in Shanghai has no service outlets as of yet.

The sales volume of EVs in a certain region is correlated with the number of $4 \mathrm{~S}$ shops in the region Han [42], and for the living standard of urban residents, their consumptiontype expenditure will have a significant impact on the number of $4 \mathrm{~S}$ cars, and the proportion of scrapped EVs in different districts to the total number of scrapped EVs is the same as the number of $4 \mathrm{~S}$ shops in different districts to the total number of $4 \mathrm{~S}$ shops. Therefore, this method is also adopted in the present study to obtain the sales volume of EVs in each district of Shanghai, as shown in Table 5.

The number of recalls is highly correlated with market ownership and annual sales volume, particularly the annual sales volume [43]. On the one hand, the recall rate reflects the number of defective vehicles, and on the other hand, it also reflects the importance that manufacturers attach to the quality of the vehicles [44]. The recall rate $R_{r}$ is the ratio of the annual number of recalled EVs $\left(N_{r}\right)$ to the annual number of EVs $\left(N_{s}\right)$ sold; that is, $R_{r}=N_{r} / N_{s}$. The average annual recall rate of EVs was determined according to Zhang and Shen [44], which shows that the average recall rate of Chinese vehicles during $2004-2017$ is $17.53 \%$. Based on this recall rate, the recall of EVs is obtained as shown in Table 6. Wu et al. [45] found that the best service life of an EVB is 5 years. As shown in Table 7, Niu [46] conducted a statistical analysis of Chinese vehicle recalls and found that the new vehicles with the highest number of recalls are those with a service life of less than 1 year, and the recall rate within 3 years is close to $80 \%$.
The recall volume during the first three years reached $77 \%$ [46], which covered most of the recall demand. The legal working days are 250 per year, as shown in Table 8, which calculates the recall volume for the next 3 years for each district in Shanghai.

Considering the special characteristics of EVs, the number of EVs recalled is an integer, and there is no fractional number of recalls. The average daily number of recalls is rounded up to solve the average daily value, and thus, there may be cases in which the average daily value multiplied by the annual working day value is greater than the total number of recalls.

Tables 9-11 show the simulated coordinate locations of each facility [15]. Table 9 shows the locations of the alternative recall sites, Table 10 shows the locations of the alternative recall processing centers, and Table 11 shows the locations of the alternative recall transfer centers. Table 12 shows the locations of the alternative echelon use centers. Table 13 shows the assignment of the parameters for each recall processing center, recall transfer center, and echelon use center.

The transport distance between points is assumed to be the Euclidean distance.

The average capacity of an EVB is approximately $500 \mathrm{kWh}$, the average weight is $500 \mathrm{~kg}$, and the value generated by each ton of EVBs is RMB 3,000 [47], and thus, the value generated by each EVB is RMB 1,500. The assignment of the recall processing center and the recall transfer center construction costs, labor costs, utilization rate of echelon use, value generated by echelon use, and maximum inventory capacity of the recall processing center are from Han [42]. The transportation cost per EVB is $12.3 \mathrm{Yuan} / \mathrm{km} \mathrm{[28].}$ Each EVB costs 0.24 Yuan/day for storage at the recall processing center, recall transfer center, and echelon use center [48]. The average packaging cost of an EVB is 232 Yuan [49]; however, when considering that the packaging is a leak-proof and corrosion-proof logistics turnover box and, according to statistics, can be reused as many as 20 times, the average single packaging cost is 11.6 Yuan. Physical inspection and functional battery testing of an EVB take 1 hour [49]. According to the data released by the Shanghai Social Security Bureau in 2019, the hourly minimum wage is 22 Yuan per hour, and thus, the cost of testing the EVB is 22 Yuan. The assignment of the transport size discount/penalty rate and transport distance discount/penalty rate is from Diabat et al. [50].

5.2. Implementation. Kronqvist et al. [51] and Wang et al. [52] presented a theoretical study and empirical analysis of LINGO solving MINLP models. An MINLP model including 822 variables and 247 constraint conditions is constructed. The optimal solution was found using Extended LINGO V18.0/DELL XPS 13. The case study shows that the maximum benefit of the three periods of the RL network for Shanghai's EVB recall is RMB 433,125,300, as shown in Table 14. 


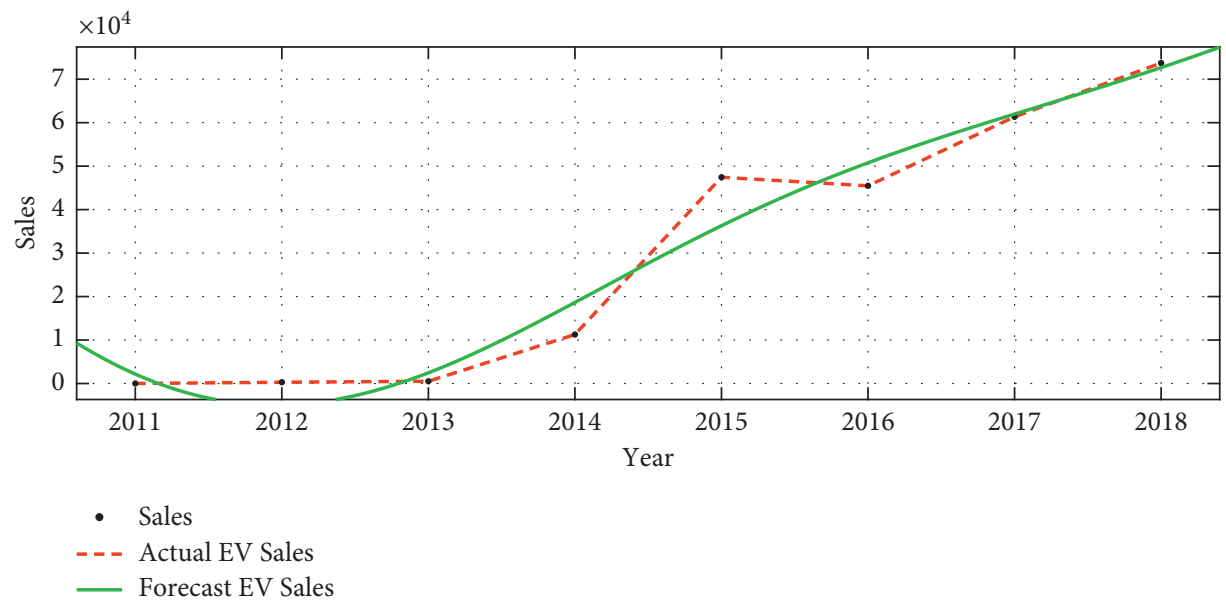

FIgURE 3: Fourier function prediction chart.

TABLE 2: EV sales in Shanghai (2019-2021).

\begin{tabular}{cc}
\hline Year & Sales \\
\hline 2019 & 82,221 \\
2020 & 94,247 \\
2021 & 101,510 \\
\hline
\end{tabular}

TABLE 3: Fourier function fit results.

\begin{tabular}{lc}
\hline Parameters & Explain \\
\hline SSE & $244,300,000$ \\
$R$-square & 0.9621 \\
Adjusted $R$-square & 0.8672 \\
RMSE & 11050 \\
\hline
\end{tabular}

TABLE 4: Quantity of EVB service outlets in each district of Shanghai.

\begin{tabular}{lcc}
\hline District & Number of service sites & $P(\%)$ \\
\hline Pudong New Area & 81 & 22.25 \\
Minhang District & 61 & 16.76 \\
Baoshang District & 44 & 12.09 \\
Jiading District & 29 & 7.97 \\
Putuo District & 29 & 7.97 \\
Songjiang District & 26 & 7.14 \\
Fengxian District & 22 & 6.04 \\
Qingpu District & 18 & 4.95 \\
Xuhui District & 17 & 4.67 \\
Yangpu District & 13 & 3.57 \\
Jing'an District & 10 & 2.75 \\
Jinshan District & 9 & 2.47 \\
Chongming District & 2 & 0.55 \\
Zhabei District & 2 & 0.55 \\
Hongkou District & 1 & 0.27 \\
\hline
\end{tabular}

The strategies for site selection for the recall reserve logistics network are shown in Table 15. The value equals 1 if a site is selected in period $p$; otherwise, it equals zero.

Table 16 shows the EVB flow distribution among the recall and recall processing centers. For instance, the number of EVBs moved from recall site 1 to recall processing center $F$ during period $1(1, \mathrm{~F}, 1)$ is 2,240 .

Table 17 shows the EVB flow distribution among the recall processing centers and recall transfer centers. For instance, the number of EVBs moved from recall processing center $C$ to recall transfer center $J$ in period $2(\mathrm{C}, \mathrm{J}, 2)$ is 4,156 .

Table 18 shows the EVB flow distribution among the recall transfer centers and echelon use centers. For instance, the number of EVBs moved from recall transfer center $G$ to echelon use center $K$ in period $3(\mathrm{G}, \mathrm{K}, 3)$ is 8,176 .

5.3. Sensitivity Analysis. This section analyzes the influence of the key parameters on the optimal value of the objective function.

The proposed multiobjective multiperiod MINLP model for the RL network of EVBs, which takes into account the recall risk, is divided into two important parts: the safety and environmental social responsibility level (SR - SET) and the economic benefit level (EB). The safety and environmental responsibility level includes minimizing the risk of social safety and environmental pollution (SET) and maximizing the social responsibility (SR). As the economic benefit (EB), an enterprise can obtain certain profits or control the loss within a certain range under the condition of ensuring safety and environmental protection.

Assuming that the parameter affecting the safety and environmental responsibility (SR - SET) is $\lambda$, the parameter affecting the economic benefit (EB)is $\mu$, the parameter affecting the social responsibility (SR) is $\varrho$, and the parameter affecting the safety and environmental response time (SET) is $\phi$. The formula is shown in

$$
\max F=\lambda(\varrho \mathrm{SR}-\phi \mathrm{SET})+\mu \mathrm{EB} .
$$

The increase or decrease in these parameters will have a certain impact on the RL network of the EVB recall, and a sensitivity analysis will help enterprise managers make reasonable recall decisions and help relevant government departments formulate reasonable policies. 
TABLe 5: Historic sales of EVs in Shanghai.

\begin{tabular}{lccccccccccc}
\hline Year & 2011 & 2012 & 2013 & 2014 & 2015 & 2016 & 2017 & 2018 & $2019 \mathrm{E}$ & $2020 \mathrm{E}$ & $2021 \mathrm{E}$ \\
\hline Pudong New Area & 3 & 66 & 114 & 2,508 & 10,557 & 10,118 & 13,651 & 16,404 & 18,294 & 20,970 & 22,586 \\
Minhang District & 2 & 50 & 86 & 1,889 & 7,952 & 7,621 & 10,283 & 12,356 & 13,780 & 15,796 & 17,013 \\
Baoshang District & 1 & 36 & 62 & 1,363 & 5,736 & 5,498 & 7,418 & 8,913 & 9,941 & 11,394 & 12,273 \\
Jiading District & 1 & 24 & 41 & 898 & 3,781 & 3,624 & 4,890 & 5,876 & 6,553 & 7,511 & 8,090 \\
Putuo District & 1 & 24 & 41 & 898 & 3,781 & 3,624 & 4,890 & 5,876 & 6,553 & 7,511 & 8,090 \\
Songjiang District & 1 & 21 & 37 & 805 & 3,388 & 3,247 & 4,381 & 5,264 & 5,871 & 6,729 & 7,248 \\
Fengxian District & 1 & 18 & 31 & 681 & 2,866 & 2,747 & 3,706 & 4,453 & 4,966 & 5,693 & 6,131 \\
Qingpu District & 1 & 15 & 25 & 558 & 2,349 & 2,251 & 3,037 & 3,649 & 4,070 & 4,665 & 5,025 \\
Xuhui District & 1 & 14 & 24 & 526 & 2,216 & 2,124 & 2,865 & 3,443 & 3,840 & 4,401 & 4,741 \\
Yangpu District & 0 & 11 & 18 & 402 & 1,694 & 1,623 & 2,190 & 2,632 & 2,935 & 3,365 & 3,624 \\
Jingan District & 0 & 8 & 14 & 310 & 1,305 & 1,251 & 1,687 & 2,027 & 2,261 & 2,592 & 2,792 \\
Jinshan District & 0 & 7 & 13 & 278 & 1,172 & 1,123 & 1,515 & 1,821 & 2,031 & 2,328 & 2,507 \\
Chongming District & 0 & 2 & 3 & 62 & 261 & 250 & 337 & 405 & 452 & 518 & 558 \\
Zhabei District & 0 & 2 & 3 & 62 & 261 & 250 & 337 & 405 & 452 & 518 & 558 \\
Hongkou District & 0 & 1 & 1 & 30 & 128 & 123 & 166 & 199 & 222 & 254 & 274 \\
Total sales volume & 12 & 298 & 514 & 11,271 & 47,446 & 45,474 & 61,354 & 73,724 & 82,221 & 94,247 & 101,510 \\
\hline
\end{tabular}

TABLE 6: Number of EVs recalled in Shanghai.

\begin{tabular}{|c|c|c|c|c|c|}
\hline Year & 2017 & 2018 & 2019 & 2020 & 2021 \\
\hline Pudong New Area & 2,393 & 2,876 & 3,207 & 3,676 & 3,959 \\
\hline Minhang District & 1,803 & 2,166 & 2,416 & 2,769 & 2,982 \\
\hline Baoshang District & 1,300 & 1,562 & 1,743 & 1,997 & 2,151 \\
\hline Jiading District & 857 & 1,030 & 1,149 & 1,317 & 1,418 \\
\hline Putuo District & 857 & 1,030 & 1,149 & 1,317 & 1,418 \\
\hline Songjiang District & 768 & 923 & 1,029 & 1,180 & 1,271 \\
\hline Fengxian District & 650 & 781 & 871 & 998 & 1,075 \\
\hline Qingpu District & 532 & 640 & 713 & 818 & 881 \\
\hline Xuhui District & 502 & 604 & 673 & 772 & 831 \\
\hline Yangpu District & 384 & 461 & 515 & 590 & 635 \\
\hline Jing'an District & 296 & 355 & 396 & 454 & 489 \\
\hline Jinshan District & 266 & 319 & 356 & 408 & 440 \\
\hline Chongming District & 59 & 71 & 79 & 91 & 98 \\
\hline Zhabei District & 59 & 71 & 79 & 91 & 98 \\
\hline Hongkou District & 29 & 35 & 39 & 45 & 48 \\
\hline Total number of recalls & 10,755 & 12,924 & 14,413 & 16,521 & 17,795 \\
\hline
\end{tabular}

TABLE 7: Age statistics of recalled cars.

\begin{tabular}{lcc}
\hline Time interval from production start to recall announcement & Number of recalls & $P(\%)$ \\
\hline Up to 1 year & 108 & 34 \\
2 years & 80 & 25 \\
$2-3$ years & 59 & 18 \\
$3-10$ years & 68 & 21 \\
More than 10 years & 5 & 2 \\
\hline
\end{tabular}

Here, we conducted a sensitivity experiment to expand or shrink each parameter to test the effect of parameter changes on the overall target. For each parameter, there are three different rates of change, that is, $0.5,1$, and 1.5 [52], as shown in the third column of Tables 19 and 20. The corresponding parameter changes for each parameter in the experimental group were $0.5,1$, and 1.5 , and the remaining four parameters were set to 1 .
Thus, there are $3 \times 2=6$ experimental instances in Table 19 and $3 \times 2=6$ experimental instances in Table 20 .

From Table 18 and Figure 4, it can be seen that the safety and environmental social responsibility parameters $\lambda$ have a small impact on the objective function results, and the economic efficiency parameter $\mu$ has a large impact. It can be seen that parameter $\lambda$ does not have a significant impact on the performance of the recall RL 
TABle 8: Predicted volume of EV recalls in Shanghai over the next 3 years.

\begin{tabular}{|c|c|c|c|c|c|c|}
\hline \multirow{2}{*}{ Year } & \multicolumn{2}{|c|}{2020} & \multicolumn{2}{|c|}{2021} & \multicolumn{2}{|c|}{2022} \\
\hline & Total & Daily average & Total & Daily average & Total & Daily average \\
\hline Pudong New Area & 2,240 & 9 & 2,569 & 11 & 2,842 & 12 \\
\hline Minhang District & 1,687 & 7 & 1,935 & 8 & 2,141 & 9 \\
\hline Baoshang District & 1,217 & 5 & 1,396 & 6 & 1,544 & 7 \\
\hline Jiading District & 802 & 4 & 920 & 4 & 1,018 & 5 \\
\hline Putuo District & 802 & 4 & 920 & 4 & 1,018 & 5 \\
\hline Songjiang District & 719 & 3 & 824 & 4 & 912 & 4 \\
\hline Fengxian District & 608 & 3 & 697 & 3 & 772 & 4 \\
\hline Qingpu District & 498 & 2 & 572 & 3 & 632 & 3 \\
\hline Xuhui District & 470 & 2 & 539 & 3 & 597 & 3 \\
\hline Yangpu District & 359 & 2 & 412 & 2 & 456 & 2 \\
\hline Jing'an District & 277 & 2 & 318 & 2 & 351 & 2 \\
\hline Jinshan District & 249 & 1 & 285 & 2 & 316 & 2 \\
\hline Chongming District & 55 & 1 & 64 & 1 & 70 & 1 \\
\hline Zhabei District & 55 & 1 & 64 & 1 & 70 & 1 \\
\hline Hongkou District & 27 & 1 & 31 & 1 & 34 & 1 \\
\hline Total number of recalls & 10,067 & 47 & 11,547 & 55 & 12,775 & 61 \\
\hline
\end{tabular}

TABLE 9: Coordinate locations of the recall sites.

\begin{tabular}{lcc}
\hline No. & $X$ & $Y$ \\
\hline 1 & 15.69 & 3.80 \\
2 & 18.67 & 24.28 \\
3 & 1.60 & 59.13 \\
4 & 9.43 & 2.27 \\
5 & 49.08 & 54.43 \\
6 & 33.14 & 10.85 \\
7 & 28.62 & 50.00 \\
8 & 24.86 & 59.39 \\
9 & 3.42 & 35.85 \\
10 & 33.23 & 21.90 \\
11 & 45.32 & 27.23 \\
12 & 46.37 & 6.36 \\
13 & 24.93 & 32.60 \\
14 & 28.07 & 33.38 \\
15 & 2.77 & 0.5 \\
\hline
\end{tabular}

TABLE 10: Alternative recall processing centers.

\begin{tabular}{lccc}
\hline No. & $X$ & $Y$ & Code \\
\hline 1 & 43.97 & 49.89 & A \\
2 & 1.57 & 12.65 & B \\
3 & 41.23 & 30.25 & C \\
4 & 5.04 & 58.97 & D \\
5 & 24.79 & 19.00 & E \\
6 & 16.18 & 20.66 & F \\
\hline
\end{tabular}

network, but parameter $\mu$ has a significant impact on the performance of the recall RL network. This obvious interest gap makes some companies in the development of recall decisions or the design of a recall RL network tend to earn maximum profit while ignoring safety issues and environmental pollution.

Parameter $\lambda$ is jointly influenced by $\varrho$ and $\phi$. Therefore, parameters $\varrho$ and $\phi$ are analyzed separately. From Figure 5, we can see that, based on the effect of the changes in parameter $\varrho$ and parameter $\phi$ on the function,
TABLE 11: Alternative recall transfer centers.

\begin{tabular}{lccc}
\hline No. & $X$ & $Y$ & Code \\
\hline 1 & 8.58 & 30.25 & $\mathrm{G}$ \\
2 & 32.36 & 28.59 & $\mathrm{H}$ \\
3 & 9.58 & 6.51 & $\mathrm{I}$ \\
4 & 47.54 & 19.31 & $\mathrm{~J}$ \\
\hline
\end{tabular}

TABLE 12: Alternative echelon use centers.

\begin{tabular}{lccc}
\hline No. & $X$ & $Y$ & Code \\
\hline 1 & 52.47 & 64.32 & $\mathrm{~K}$ \\
2 & 77.56 & 78.71 & $\mathrm{~L}$ \\
\hline
\end{tabular}

parameter $\varrho$ plays a positive correlated role, and parameter $\phi$ plays a negatively correlated role. The greater the social responsibility, the more obvious the increase in the target function; in addition, the higher the requirements for safety and environmental protection as well as the recall response time, the more obvious the decrease in the target function.

The recall of EVBs is unique owing to its characteristics, such as the existence of certain safety risks, potential environmental pollution, and significant economic benefits. Therefore, while countries are encouraging the recycling or recall of EVBs, they have also issued relevant laws, regulations, or standards, and developed a rigorous and safe recall process. Although there is huge interest in EVBs, we cannot neglect safety and environmental protection and strive for unrestricted profits. This is due to the irreplaceable nature of safety and environmental protection and the weak economics of recalls, requiring policymakers and business managers to rely on standard operations and scientific management to gain appropriate benefits and implement an extended producer responsibility system to actively assume their social responsibility 
Table 13: Parameter assignments.

\begin{tabular}{|c|c|c|c|}
\hline Content & Parameter & Value & Unit of measurement \\
\hline Construction cost of recall processing center & $f i_{s}^{p}$ & $10,000,000$ & Yuan \\
\hline Construction cost of recall transfer center & $f i_{t}^{p}$ & $8,000,000$ & Yuan \\
\hline Construction cost of echelon use center & $f i_{e}^{p}$ & $12,000,000$ & Yuan \\
\hline Jobs created in a recall processing center & $j c_{s}^{p}$ & 30 & No. of people \\
\hline Jobs created in a recall transfer center & $j c_{t}^{p}$ & 45 & No. of people \\
\hline Jobs created in an echelon use center & $j c_{e}^{p}$ & 100 & No. of people \\
\hline Transportation costs per EVB & $s c$ & 12.3 & Yuan \\
\hline Packaging cost per EVB & $p k c$ & 11.6 & Yuan \\
\hline Testing cost per EVB & $d c$ & 22 & Yuan \\
\hline Daily labor cost per employee & $l c$ & 200 & Yuan \\
\hline Storage cost per unit per day at recall processing center & $w c_{s}$ & 0.24 & Yuan \\
\hline Storage cost per unit per day at recall transfer center & $w c_{t}$ & 0.24 & Yuan \\
\hline Storage cost per unit per day at echelon use center & $w c_{e}$ & 0.24 & Yuan \\
\hline Maximum processing capacity of recall processing center & $M_{s}$ & 800 & No. of items \\
\hline Maximum processing capacity of recall transfer center & $M_{t}$ & 800 & No. of items \\
\hline Maximum processing capacity of echelon use center & $M_{e}$ & 1,500 & No. of items \\
\hline Maximum inventory capacity of recall processing center & $W_{s}$ & 800 & No. of items \\
\hline Maximum inventory capacity of recall transfer center & $W_{t}^{s}$ & 800 & No. of items \\
\hline \multirow[t]{3}{*}{ Maximum inventory capacity of echelon use center } & $W_{e}$ & 2,000 & No. of items \\
\hline & $\delta_{0}^{e}$ & 1 & - \\
\hline & $\delta_{1}$ & 0.8 & - \\
\hline \multirow[t]{5}{*}{ Transport size discount rate } & $\delta_{2}$ & 0.6 & - \\
\hline & $k_{1}$ & 200 & No. of items \\
\hline & $k_{2}$ & 400 & No. of items \\
\hline & $\gamma_{0}$ & 1 & - \\
\hline & $\gamma_{1}$ & 1.1 & - \\
\hline \multirow[t]{3}{*}{ Transport distance penalty rate } & $\gamma_{2}$ & 1.2 & - \\
\hline & $g_{1}$ & 25 & $\mathrm{~km}$ \\
\hline & $g_{2}$ & 60 & $\mathrm{~km}$ \\
\hline Value of EVBs after echelon use & $\operatorname{Pr}$ & 1,500 & Yuan/each \\
\hline Ratio of echelon use & $\beta$ & 0.9 & Percentage (\%) \\
\hline
\end{tabular}

TABle 14: Solutions.

\begin{tabular}{lc}
\hline Optional solution found & Profit (Yuan) \\
\hline Objective value & $433,125,300$ \\
Objective bound & $433,125,300$ \\
Extended solver steps & 31 \\
Total solver iterations & 14,039 \\
Elapsed runtime seconds & 11.07 \\
Model class & MINLP \\
Solver type & Global \\
\hline
\end{tabular}

TABle 15: Site selection scheme.

\begin{tabular}{lcccccccccccc}
\hline Period & \multicolumn{11}{c}{ RL facilities } \\
\hline \multirow{2}{*}{$p=1$} & A & B & C & D & E & F & G & H & I & J & K & L \\
& 0 & 0 & 1 & 1 & 0 & 1 & 1 & 0 & 0 & 1 & 1 & 0 \\
& A & B & C & D & E & F & G & H & I & J & K & L \\
& 0 & 0 & 1 & 1 & 0 & 1 & 1 & 0 & 0 & 1 & 1 & 0 \\
& A & B & C & D & E & F & G & H & I & J & K & L \\
& 0 & 0 & 1 & 1 & 0 & 1 & 1 & 0 & 0 & 1 & 1 & 0 \\
\hline
\end{tabular}

under the premise of safety and environmental protection. In the development of recall-related policies or standards, we cannot rely on the economic benefits of a single
TABle 16: Traffic distribution from recall site to recall processing center.

\begin{tabular}{lccccc}
\hline Traffic & Value & Traffic & Value & Traffic & Value \\
\hline$(1, \mathrm{~F}, 1)$ & 2,240 & $(1, \mathrm{~F}, 2)$ & 2,569 & $(1, \mathrm{~F}, 3)$ & 2,842 \\
$(2, \mathrm{~F}, 1)$ & 1,687 & $(2, \mathrm{~F}, 2)$ & 1,935 & $(2, \mathrm{~F}, 3)$ & 2,141 \\
$(3, \mathrm{D}, 1)$ & 1,217 & $(3, \mathrm{D}, 2)$ & 1,396 & $(3, \mathrm{D}, 3)$ & 1,544 \\
$(4, \mathrm{~F}, 1)$ & 802 & $(4, \mathrm{~F}, 2)$ & 920 & $(4, \mathrm{~F}, 3)$ & 1,018 \\
$(5, \mathrm{C}, 1)$ & 802 & $(5, \mathrm{C}, 2)$ & 920 & $(5, \mathrm{C}, 3)$ & 1,018 \\
$(6, \mathrm{C}, 1)$ & 719 & $(6, \mathrm{C}, 2)$ & 824 & $(6, \mathrm{C}, 3)$ & 912 \\
$(7, \mathrm{C}, 1)$ & 608 & $(7, \mathrm{C}, 2)$ & 697 & $(7, \mathrm{C}, 3)$ & 772 \\
$(8, \mathrm{C}, 1)$ & 498 & $(8, \mathrm{C}, 2)$ & 572 & $(8, \mathrm{C}, 3)$ & 632 \\
$(9, \mathrm{~F}, 1)$ & 470 & $(9, \mathrm{~F}, 2)$ & 539 & $(9, \mathrm{~F}, 3)$ & 597 \\
$(10, \mathrm{C}, 1)$ & 359 & $(10, \mathrm{C}, 2)$ & 412 & $(10, \mathrm{C}, 3)$ & 456 \\
$(11, \mathrm{C}, 1)$ & 277 & $(11, \mathrm{C}, 2)$ & 318 & $(11, \mathrm{C}, 3)$ & 351 \\
$(12, \mathrm{C}, 1)$ & 249 & $(12, \mathrm{C}, 2)$ & 285 & $(12, \mathrm{C}, 3)$ & 316 \\
$(13, \mathrm{C}, 1)$ & 55 & $(13, \mathrm{C}, 2)$ & 64 & $(13, \mathrm{C}, 3)$ & 70 \\
$(14, \mathrm{C}, 1)$ & 55 & $(14, \mathrm{C}, 2)$ & 64 & $(14, \mathrm{C}, 3)$ & 70 \\
$(15, \mathrm{~F}, 1)$ & 27 & $(15, \mathrm{~F}, 2)$ & 3 & $(15, \mathrm{~F}, 3)$ & 34 \\
\hline
\end{tabular}

indicator and need to fully consider the social safety and environmental aspects of the community as well as other factors to ensure that an EVB recall can be safe, fast, and green and achieve reusability. 
TABLE 17: Traffic distribution from recall processing center to recall transfer center.

\begin{tabular}{lccccc}
\hline Traffic & Value & Traffic & Value & Traffic & Value \\
\hline (C, J, 1) & 3,622 & $(\mathrm{C}, \mathrm{J}, 2)$ & 4,156 & $(\mathrm{C}, \mathrm{J}, 3)$ & 4,597 \\
$(\mathrm{D}, \mathrm{G}, 1)$ & 1,217 & $(\mathrm{D}, \mathrm{G}, 2)$ & 1,396 & $(\mathrm{D}, \mathrm{G}, 3)$ & 1,544 \\
(F, G, 1) & 5,226 & $(\mathrm{~F}, \mathrm{G}, 2)$ & 5,966 & $(\mathrm{~F}, \mathrm{G}, 3)$ & 6,632 \\
\hline
\end{tabular}

TABLE 18: Traffic distribution from recall transfer center to echelon use center.

\begin{tabular}{lccccc}
\hline Traffic & Value & Traffic & Value & Traffic & Value \\
\hline$(\mathrm{G}, \mathrm{K}, 1)$ & 6,443 & $(\mathrm{G}, \mathrm{K}, 2)$ & 7,362 & $(\mathrm{G}, \mathrm{K}, 3)$ & 8,176 \\
$(\mathrm{~J}, \mathrm{~K}, 1)$ & 3,622 & $(\mathrm{~J}, \mathrm{~K}, 2)$ & 4,156 & $(\mathrm{~J}, \mathrm{~K}, 3)$ & 4,597 \\
\hline
\end{tabular}

TABLE 19: Values of parameters $\lambda$ and $\mu$ under different rates of change.

\begin{tabular}{lccccc}
\hline Parameters & Instance no. & Change rate & $\lambda$ & $\mu$ & Value \\
\hline \multirow{3}{*}{$\lambda$, SR - SET } & 1 & 0.5 & $\mathbf{0 . 5}$ & 1 & $433,128,000$ \\
& 2 & 1 & $\mathbf{1}$ & 1 & $433,126,300$ \\
& 3 & 1.5 & $\mathbf{1 . 5}$ & 1 & $433,124,600$ \\
$\mu, \mathrm{EB}$ & 4 & 0.5 & 1 & $\mathbf{0 . 5}$ & $216,561,500$ \\
& 5 & 1 & 1 & $\mathbf{1}$ & $433,126,300$ \\
& 6 & 1.5 & 1 & $\mathbf{1 . 5}$ & $649,691,200$ \\
\hline
\end{tabular}

TABLE 20: The values of parameters $\varrho$ and $\phi$ under different change rates.

\begin{tabular}{lcccccc}
\hline Parameters & Instance no. & Change rate & $\varrho$ & $\phi$ & $\mu$ & Value \\
\hline \multirow{3}{*}{ @ SR } & 1 & 0.5 & $\mathbf{0 . 5}$ & 1 & 1 & $433,124,800$ \\
& 2 & 1 & $\mathbf{1}$ & 1 & 1 & $433,125,300$ \\
& 3 & 1.5 & $\mathbf{1 . 5}$ & 1 & 1 & $433,125,700$ \\
\hline \multirow{3}{*}{$\phi$, SET } & 4 & 0.5 & 1 & $\mathbf{0 . 5}$ & 1 & $433,127,900$ \\
& 5 & 1 & 1 & $\mathbf{1}$ & 1 & $433,125,300$ \\
& 6 & 1.5 & 1 & $\mathbf{1 . 5}$ & 1 & $433,122,600$ \\
\hline
\end{tabular}

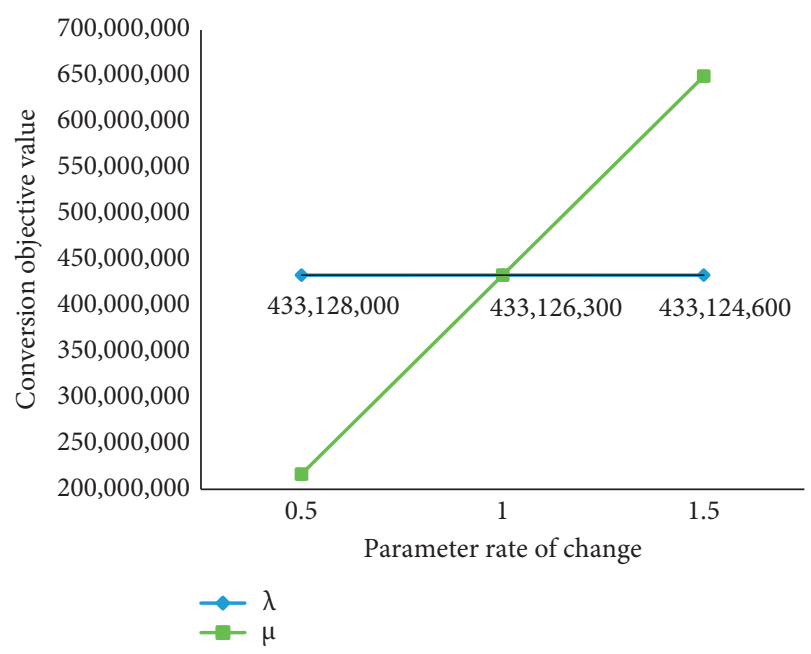

FIGURE 4: The influence of the change in parameters $\lambda$ and $\mu$ on the optimal value of the objective function.

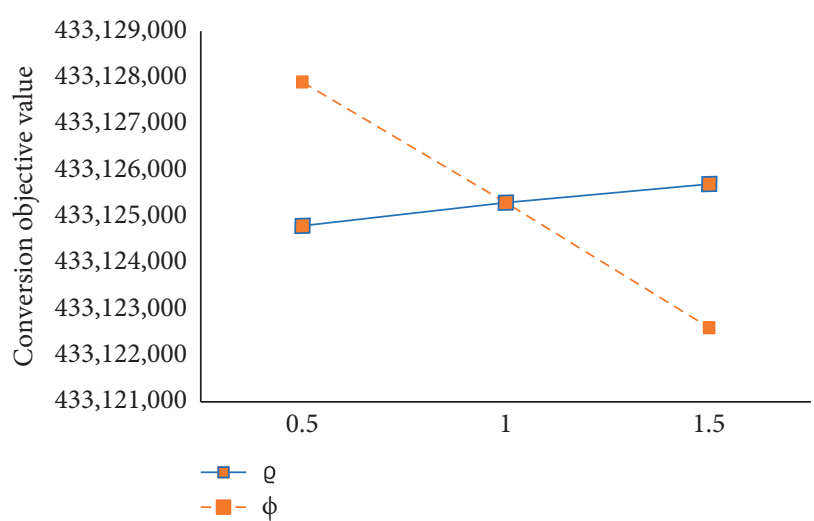

Figure 5: Influence of the change in parameters $\varrho$ and $\phi$ on the optimal value of the objective function.

\section{Conclusion and Future Research}

This study aims to solve the problem of optimizing the configuration of RL network facilities for an EVB recall, which is not only a key issue of EVB safety risk management but also an inevitable requirement in promoting the construction of an ecological and green society. First, a multiobjective and multiperiod dynamic model is established to minimize safety and environmental risks, maximize social responsibility and economic benefits, and achieve an RL infrastructure of an EVB recall, including the service outlet, recall site, recall processing center, recall transfer center, and echelon use center. Second, this study focused on the recall of EVBs from Shanghai. Based on historical data and a Fourier fitting, the number of recalled EVBs is predicted for the next 3 years. In this study, based on the locations of these potential RL facilities and the distribution of RL flows in Shanghai's EVB recall, an RL network for each period was considered. Finally, the impact of the weighting of the three factors of safety and environmental protection, social responsibility, and economic benefits on the location decision of a recall RL network was investigated.

The research results are summarized as follows: (1) A multiobjective and multiperiod dynamic site selection model was constructed by combining prediction and MINLP optimization methods, which were used to determine the site selection of each facility and the distribution of the EVB RL network. (2) An EVB recall RL model with considerations regarding safety and the environment was developed, and the synergistic development of safety and environmental protection, social responsibility, and economic benefits was achieved. (3) A sensitivity analysis of the parameters used in the recall RL network was conducted to test the proposed factors that affect the network performance. Although the degree of influence of economic benefit in an objective function is much larger than the degree of influence of safety, environmental protection, and social responsibility, without safety, all economic benefits are zero, and without environmental protection, the economic benefits are greatly discounted.

A study on the design of an RL network for EVBs considering the risk of a recall has helped focus attention on 
the management of RL of EVBs. This study uses a combination of predictive techniques and MINLP modeling and considers safety, environmental protection, social responsibility, and economic benefits, which may improve the planning, operation, and management of the RL network for EVB recalls. Furthermore, this study provides possible solutions to maintain the balance between safety, environmental protection, social responsibility, and economic efficiency and also provides a reference for decision-makers to prioritize different factors in different recall situations. This study provides some insight into how to scientifically and effectively design an RL network for the recall of products with certain safety risks or environmental pollution hazards, such as EVBs.

However, owing to the complexity of the RL network and consumer uncertainty, future research still needs to be conducted to improve and supplement this field. In this study, the details between different recalled batteries have not been fully considered, such as the time and cost from vehicle owners to service outlets, the uncertainty in vehicle running time Zhen [53] and EVBs recall time, and the cost of purchasing testing equipment. Therefore, future research will consider the details and quantify the study. Although there are still some shortcomings in this study, it was demonstrated that when there are significant potential benefits and risks, managers should give priority to safety and environmental protection and establish relevant mandatory or guiding systems or standards to properly restrain the behavior of different enterprises. However, policymakers should also provide certain incentives (tax breaks, etc.) to encourage companies to consider safety and environmental protection and to encourage companies and industries to make technological improvements or industrial upgrades. This is of positive significance for building a safe, green, and sustainable ecological society.

\section{Data Availability}

The data used to support the findings of this study are available from the corresponding author upon request.

\section{Conflicts of Interest}

The authors declare that they have no conflicts of interest.

\section{Acknowledgments}

This research was supported by the National Social Science Foundation of China (20BGL200) "Research on the Construction and Governance of EVB Recycling Ecosystem under the Background of Block-Chain"; Key Soft Science Project of Shanghai Science and Technology Commission (19692107700), "Research on the Recycling Mechanism of EVB Based on Urban Safety and Stepwise Utilization"; Research Project of Humanities and Social Sciences of Ministry of Education, "Research on Credit Rating Innovation of Small and Micro Enterprises Based on Environmental Behavior Indicators and Internet Finance Indicators" (15YJAZH121); Shanghai Highland Course-Environmental
Science and Engineering (Center for Resource Recycling Science and Engineering, Shanghai Polytechnic University), "Reverse Logistics Subject" (A30DB182602); Shanghai Polytechnic University Management Science and Engineering Discipline Construction Fund "Logistics Management Project" (XXKPY1606); Graduate Education and Research Project of Shanghai Polytechnic University (A01GY19H010-j01); and Construction of Pilot Major with Logistics Management for Connecting Secondary Vocational and Undergraduate Courses in Shanghai Polytechnic University.

\section{References}

[1] IEA, Global EV Outlook, International Energy Agency, Paris, France, 2020.

[2] SAMR, Notice from the State Administration of Market Regulation on the National Recall of Automobiles and Consumer Products in 2019, SAMR, Beijing, China, 2020.

[3] NDANEV, "Electricvehicles national regulatory platform big data safety supervision results report," 2019.

[4] Y. A. Alamerew and D. Brissaud, "Modelling reverse supply chain through system dynamics for realizing the transition towards the circular economy: a case study on electric vehicle batteries," Journal of Cleaner Production, vol. 254, Article ID 120025, 2020.

[5] J. García, E. Jadraque Gago, and A. Girard, "Processes and technologies for the recycling and recovery of spent lithiumion batteries," Renewable and Sustainable Energy Reviews, vol. 60, pp. 195-205, 2016.

[6] J. R. Stock, Reverse Logistics: White Paper, Council of Logistics Management, Oak Brook, IL, USA, 1992.

[7] J. Sarkis, M. M. Helms, and A. A. Hervani, "Reverse logistics and social sustainability," Corporate Social Responsibility and Environmental Management, vol. 17, no. 6, pp. 337-354, 2010.

[8] H. Hao, Z. Wang, H. Lin, Q. Zhang, M. Huang, and J. Zhu, "Fifth profit source: commercial value and mode of reverse logistics in China," Logistics Technology, vol. 36, no. 8, pp. 47-50, 2017.

[9] Z. Wang, H. Hao, F. Gao, Q. Zhang, J. Zhang, and Y. Zhou, "Multi-attribute decision making on reverse logistics based on DEA-TOPSIS: a study of the Shanghai end-of-life vehicles industry," Journal of Cleaner Production, vol. 214, 2019.

[10] M. Fleischmann, P. Beullens, J. Bloemhof-Ruwaard, and L. Van Wassenhove, "The impact of product recovery on logistics network design," Production and Operations Management, vol. 10, pp. 156-173, 2001.

[11] A. I. Barros, R. Dekker, and V. Scholten, "A two-level network for recycling sand: a case study," European Journal of Operational Research, vol. 110, no. 2, pp. 199-214, 1998.

[12] H. Min, H. Jeung Ko, and C. Seong Ko, “A genetic algorithm approach to developing the multi-echelon reverse logistics network for product returns," Omega, vol. 34, no. 1, pp. 56-69, 2006.

[13] G. Zhou and Z. Cao, "Research on the application of genetic algorithm in the location problem of reverse logistics network," Chinese Journal of Management Science, vol. 1, pp. 43-48, 2005.

[14] N. Zaarour, "A reverse logistics network model for handling returned products," International Journal of Engineering Business Management, vol. 6, pp. 6-13, 2014.

[15] X. Zhou, Y. Gao, J. Ren, and H. Guo, "Multi-objective location model and algorithm for remanufacturing reverse logistics 
under government subsidies," Systems Engineering-Theory \& Practice, vol. 35, pp. 1996-2003, 2015.

[16] L. Zhen, "A bi-objective model on multiperiod green supply chain network design," IEEE Transactions on Systems, Man, and Cybernetics: Systems, vol. 50, no. 3, pp. 771-784, 2020.

[17] K. Govindan, P. Paam, and A.-R. Abtahi, "A fuzzy multiobjective optimization model for sustainable reverse logistics network design," Ecological Indicators, vol. 67, pp. 753-768, 2016.

[18] J. Trochu, A. Chaabane, and M. Ouhimmou, "Reverse logistics network redesign under uncertainty for wood waste in the CRD industry," Resources, Conservation and Recycling, vol. 128 , pp. 32-47, 2018.

[19] R. K. Nageswara, A. Kumar, J. Sarkis, and M. Tiwari, "Effect of carbon tax on reverse logistics network design," Computers and Industrial Engineering, vol. 139, Article ID 106184, 2019.

[20] C. Hoyer, K. Kieckhäfer, and T. S. Spengler, "A strategic framework for the design of recycling networks for lithiumion batteries from electric vehicles," Glocalized Solutions for Sustainability in Manufacturing, Springer, Berlin, Germany, pp. 79-84, 2011.

[21] H. Hao, Q. Zhang, and Z. Wang, "Feasibility evaluation of reverse logistics of scrapped automobile battery-based on improved fuzzy neural network," China Business and Market, vol. 32, pp. 15-24, 2018.

[22] H. Huo, Y. Xing, M. Pecht, B. J. Züger, N. Khare, and A. Vezzini, "Safety requirements for transportation of lithium batteries," Energies, vol. 10, no. 6, p. 793, 2017.

[23] S. Sloop, L. Crandon, and M. Allen, "A direct recycling case study from a lithium-ion battery recall," Sustainable Materials and Technologies, vol. 25, Article ID e00152, 2020.

[24] I. Hakim, A. Danielle, B. Claude, and R. Sophie, Designing a Sustainable Recycling Network for Batteries from Electric Vehicles. Development and Optimization of Scenarios, Springer, Berlin, Germany, 2013.

[25] X. Wang, G. Gaustad, C. W. Babbitt, and K. Richa, "Economies of scale for future lithium-ion battery recycling infrastructure," Resources, Conservation and Recycling, vol. 83, pp. 53-62, 2014.

[26] A. Jayant, P. Gupta, and S. K. Garg, "Reverse logistics network design for spent batteries: a simulation study," International Journal of Logistics Systems and Management, vol. 18, no. 3, pp. 343-365, 2014.

[27] T. P. Hendrickson, O. Kavvada, N. Shah, R. Sathre, and C. Scown, "Life-cycle implications and supply chain logistics of electric vehicle battery recycling in California," Environmental Research Letters, vol. 10, no. 1, Article ID 014011, 2015.

[28] M. Tadaros, A. Migdalas, B. Samuelsson, and A. Segerstedt, "Location of facilities and network design for reverse logistics of lithium-ion batteries in Sweden," Operational Research, vol. 8, pp. 1-21, 2020.

[29] I. Masudin, T. E. Saputro, G. Arasy, and F. Jie, "Reverse logistics modeling considering environmental and manufacturing costs: a case study of battery recycling in Indonesia," International Journal of Technology, vol. 10, no. 1, p. 189, 2019.

[30] J. Wang, H. Li, H. Lu, H. Yang, and C. Wang, "Integrating offline logistics and online system to recycle e-bicycle battery in China," Journal of Cleaner Production, vol. 247, Article ID 119095, 2020.

[31] U. Akkucuk and Y. Gencer, "Product recalls as an important category of reverse logistics," 2016.

[32] V. Jayaraman, R. A. Patterson, and E. Rolland, "The design of reverse distribution networks: models and solution procedures," European Journal of Operational Research, vol. 150, no. 1, pp. 128-149, 2003.

[33] H.-T. Hu, Y.-H. Ru, R.-J. Wang, and Y.-K. Wang, "Dynamic closed-loop supply chain network design based on recall and return of goods," in Proceedings of the 2010 International Conference on Logistics Systems and Intelligent Management (ICLSIM), pp. 788-791, Harbin, China, January 2010.

[34] J. Chen, J. Ji, and X. Bao, "Dual-objective emergency recall closed-loop network planning considering time factors," Control and Decision, vol. 25, pp. 975-980, 2010, In Chinese.

[35] W. Pan, J. Wang, and S. Wang, "Research on location selection of food recall center based on improved genetic algorithm," Logistics Engineering and Management, vol. 36, pp. 53-55, 2014, In Chinese.

[36] L. Gao, Research on Logistics Network Operation of Drug Recall, Beijing Jiaotong University, Beijing, China, 2011.

[37] Y. Huang and L. Wang, "Design of medicine reverse logistics network based on problem drug recall," Value Engineering, vol. 36, pp. 71-72, 2017, In Chinese.

[38] L. Zhen, "Modeling of yard congestion and optimization of yard template in container ports," Transportation Research Part B: Methodological, vol. 90, pp. 83-104, 2016.

[39] A. Fumi, A. Pepe, L. Scarabotti, and M. Schiraldi, "Fourier analysis for demand forecasting in a fashion company," International Journal of Engineering Business Management, vol. 5, 2013.

[40] B. A. Sadek, E. W. Martin, and S. A. Shaheen, "Forecasting truck parking using fourier transformations," Journal of Transportation Engineering, Part A: Systems, vol. 146, no. 8, 2020.

[41] H. Hao, Q. Zhang, Z. Wang, and J. Zhang, "Forecasting the number of end-of-life vehicles using a hybrid model based on grey model and artificial neural network," Journal of Cleaner Production, vol. 202, 2018.

[42] N. Han, Research on the Optimization of Reverse Logistics Network of Waste Electric Vehicle Battery in City A, Donghua University, Shanghai, China, 2019.

[43] Y. Chen, C. Liu, and J. Zhang, "The management mechanism of China's defective automobile product recall: current status and development," Journal of Automotive Safety and Energy, vol. 6, pp. 119-127, 2015.

[44] J. Zhang and H. Shen, "The impact of China's defective car recall on car sales," Enterprise Economy, vol. 4, pp. 101-109, 2020.

[45] X. Wu, J. Wang, W. Tian, and Z. Zuo, "Safety strategy for echelon utilization of retired batteries based on application requirements," Energy Storage Science and Technology, vol. 7, pp. 1094-1104, 2018.

[46] M. Niu, Discussion and Research on China's Automobile Recall System, Changan University, Xi'an, China, 2011.

[47] X. Ma, Y. Ma, J. Zhou, and S. Xiong, "The recycling of spent power battery: economic benefits and policy suggestions," IOP Conference Series: Earth and Environmental Science, vol. 159, Article ID 012017, 2018.

[48] M. Li, Research on the Construction of Reverse Logistics Network for Used Electric Vehicle Battery under Uncertain Environment, Master's thesis, Donghua University, Shanghai, China, 2014.

[49] L. Canals Casals, B. Amante García, and M. M. González Benítez, "A cost analysis of electric vehicle batteries second life businesses," in Proceedings of the XVIII Congreso Internacional de Dirección e Ingeniería de Proyectos, pp. 129-141, Springer, Alcañiz, Spain, 2016. 
[50] A. Diabat, D. Kannan, M. Kaliyan, and D. Svetinovic, "An optimization model for product returns using genetic algorithms and artificial immune system," Resources, Conservation and Recycling, vol. 74, pp. 156-169, 2013.

[51] J. Kronqvist, D. E. Bernal, A. Lundell, and I. E. Grossmann, “A review and comparison of solvers for convex MINLP," Optimization and Engineering, vol. 20, no. 2, pp. 397-455, 2019.

[52] Z. Wang, L. Huang, and C. He, "A multi-objective and multiperiod optimization model for urban healthcare waste's reverse logistics network design," Journal of Combinatorial Optimization, pp. 1-28, 2019.

[53] L. Zhen, "Tactical berth allocation under uncertainty," European Journal of Operational Research, vol. 247, no. 3, pp. 928-944, 2015. 